\title{
Redox Reactions in An YSZ/Pt Interface- An In-situ Electron Holography and EELS Study
}

\author{
A.H. Tavabi, ${ }^{*}$ T. Tanji, $* *$ \\ * Graduate School of Engineering, Nagoya University, Furo-cho, Chikusa, Nagoya 464-8603, \\ Japan \\ ** Ecotopia Science Institute, Nagoya University, Furo-cho, Chikusa, Nagoya 464-8603, Japan
}

Yttria stabilized zirconia (YSZ)/Pt interface is the basic system of solid oxide fuel cells with the superior performance. Named three phase boundary, when combined with oxygen, it would determine the final efficiency of cell operation. In recent years, this interface has been investigated on characteristics [1] and electronic structure [2] and in reaction with oxygen [3]. In the present research we have applied in situ EELS and electron holography at intermediate temperatures and in oxygen atmosphere to study initial states of redox reactions in this system.

A hetero-interface YSZ/Pt bulk sample was prepared on a single crystal YSZ (100) with 13 mol $\% \mathrm{Y}_{2} \mathrm{O}_{3}$ and a $\mathrm{Pt}$ thin layer was deposited by pulsed laser deposition method. Cross section specimens were prepared by FIB (Hitachi FB2100) by micro-sampling technique. In-situ electron holography observations were performed with a field emission Hitachi HF2000 electron microscope which equipped with an electron biprism. An open type in-situ system was installed on this microscope to insert oxygen directly in the specimen area. A FIB thinned specimen was mounted on the edge of a tantalum ribbon and milled in the direction that interface was placed at the top and then another region of YSZ substrate was milled by tilting technique at $4 \mu \mathrm{m}$ distance with interface, named edge area (fig. 1). Finally, the tantalum ribbon was mounted on a special TEM holder which is designed for high temperatures observation in the gas atmosphere. In-situ EELS studies were accomplished by high voltage JEM 1000K RS, recently installed in Nagoya University. This microscope has been equipped with a Gatan Imaging Filter to perform EELS analysis. Another cross section specimen was prepared by the same micro sampling FIB technique and mounted on a conventional FIB grid. At this step, two other regions of substrate, far from platinum interface, were thinned and marked to be able to compare with interface area during in-situ observations.

In-situ electron holography was preformed from room temperature to $400^{\circ} \mathrm{C}$ by $100^{\circ} \mathrm{C}$ steps. At each step both interface and edge areas were observed. Phase images were reconstructed from acquired holograms and phase profiles were plotted from both regions. Results showed a considerable decrease in phase shift amount in YSZ during heating process that imply on oxygen ions migration from the observed area. It was found that oxygen transferred at a considerably higher rate in the interface region compare with the area far from the interface. After oxygen exposure to the specimen at $400^{\circ} \mathrm{C}$, it was found out that phase shifts in both regions suddenly increase even at a very low pressure of oxygen. Supposedly, changes are significantly higher in interface region. These results show that oxygen reduction reaction has occurred at interface, so that $\mathrm{O}_{2}$ molecules were adsorbed through platinum, reduced and diffused into YSZ.

EELS spectra form Pt interface area and two other regions of the YSZ substrate far from interface acquired at room temperature, $100^{\circ} \mathrm{C}, 300^{\circ} \mathrm{C}$ and $500^{\circ} \mathrm{C}$, denoted on the similar 
phenomena in agreement with electron holography results. At room temperature all three regions similarly showed typical zirconia structure. However, by increasing the temperature to $300^{\circ} \mathrm{C}$, a reversal in the intensities of $\mathrm{Zr} \mathrm{L} \mathrm{L}_{2}$ and $\mathrm{L}_{3}$ lines was observed at the vicinity of platinum (Fig. 2) that may indicate a transition in $\mathrm{Zr}$ oxidation stat from $4+$ to $3+$. Reasonably, any significant change was not found in the regions far from the platinum interface. This shows that at the interface area, zirconium valance state has changed from $\mathrm{Zr}+4$ to $\mathrm{Zr}+3$ due to platinum catalytic behavior and oxygen ions removed from the zirconia into gas state.

\section{References}

[1] T. Tanji et al. Microsc Microanal, 15 (2009) 1424-25

[2] V. Srot et al. Solid State Ionics, 181 (2010) 1616-1622

[3] H. Huang et al. J. Electrochem. Soc., 156 (2009) B392-B396.

[4] Authors gratefully acknowledge Dr. Kazou Yamamoto and Dr. Takeharu Kato at Japan Fine Ceramic Center (JFCC) for fruitful discussions and FIB facility. Dr. Shigeo Arai at Ecotopia Science Institute, Nagoya University is highly appreciated for valuable helps on EELS Measurements. The present work was partially supported by the Grant-in-Aid for Scientific Research on Priority Area, "Nonionics" (439), and No. 20246013 by the Ministry of Education, Culture, Sports, and Technology, Japan.
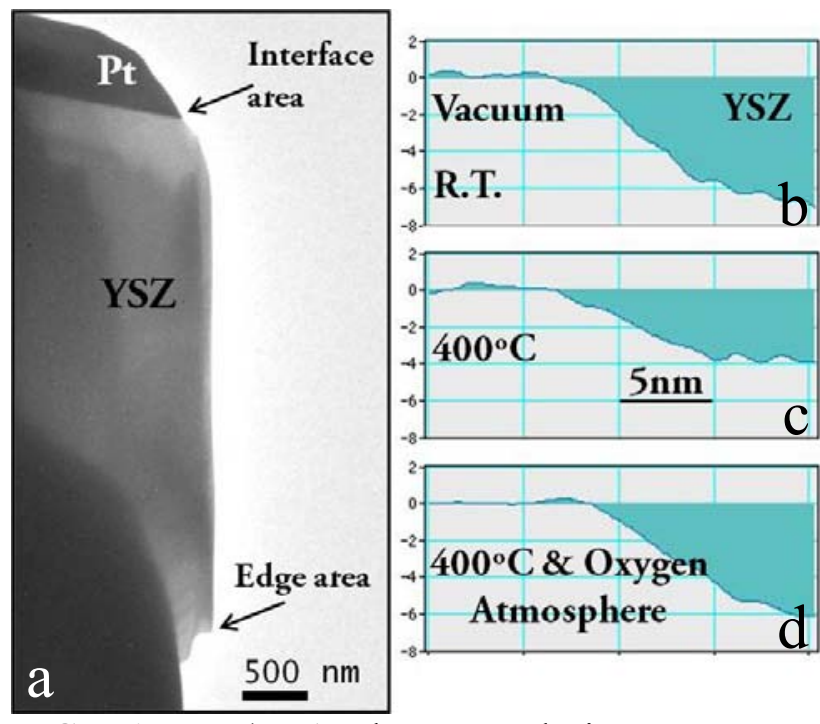

FIG. 1 .

a) A low

resolution TEM micrograph of FIB cross sectioned specimen. b-d) phase shift profiles of interface area at R.T., $400^{\circ} \mathrm{C}$ and $400^{\circ} \mathrm{C}$ in oxygen exposure.
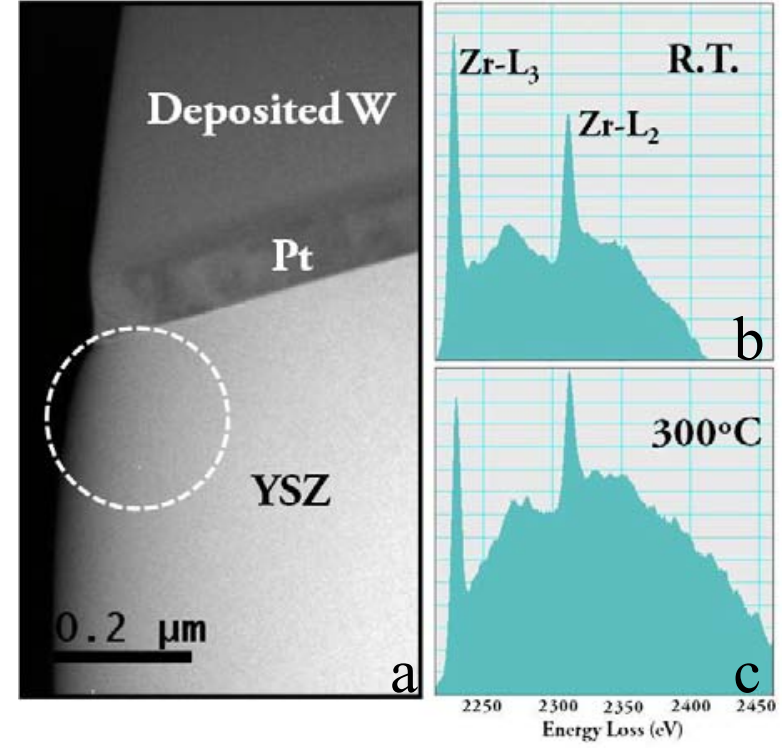

FIG. 2. a) An energy filtered image of O-K post-edge, (dashed line shows aperture position to acquire EELS spectra. b\&c) energy-loss spectrum of zirconia recorded at R.T. and $300^{\circ} \mathrm{C}$, respectively. 